\title{
Negative Sequence-Based Schemes for Power System Protection - Review and Challenges
}

\author{
Safa Kareem Al-Sachit \\ Electrical and Computer Engineering, \\ University of Auckland \\ Auckland, New Zealand \\ slas931@aucklanduni.ac.nz
}

\author{
Mohammad Javad Sanjari \\ Electrical and Computer Engineering, \\ University of Auckland \\ Auckland, New Zealand \\ msan310@aucklanduni.ac.nz
}

\author{
Nirmal-Kumar C Nair \\ Electrical and Computer Engineering, \\ University of Auckland \\ Auckland, New Zealand \\ n.nair@auckland.ac.nz
}

\begin{abstract}
This paper presents a review of the negative sequence-based protection relays development and their applications on electrical power networks and discusses the related challenges. Recent power system requires selective, reliable, rapid fault detection and clearance mechanisms especially for the transmission line networks, which are highly exposed to environmental incidents. Most of the negative sequence protection techniques and recent challenges are discussed in this paper.
\end{abstract}

Keywords-Negative Sequence Relays, Relays, Power System Protection, Transmission Line Protection.

\section{INTRODUCTION}

$\mathrm{T}$ he continued development of the power system makes it very complex and challenging where the network has become more demanding for reliability, and a high level of power quality. Developments like smart grid and microgrid have led to the introduction of new guides and standards for control and protection to reduce undesirable power system failures. Power system instability can be initiated by simple faults or by high load. This can result in power oscillations or islanding [1]. As a result, many techniques have been used to protect the power system and keep it operating with a balanced three-phase sinusoidal at steady-state condition. There are some events that can cause unbalanced operations including trees in contact with a conductor, a lightning strike, or pendulous power line. This unbalanced system was complicated to analyze until 1913, when Fortescue developed a new theory based on analyzing each of the unbalanced three-phase voltage or current systems into their symmetrical components. This theory was used to solve and understand the unbalanced operation conditions of a normally balanced system and the influence of voltage and current harmonics [2].

There are three symmetrical components for each voltage or current phasor, i.e. positive, negative, and zero sequence components. They are mathematical components representing the actual voltage and current in unbalanced systems. These components represent the order of rotation of any of the three phases [3].

Protection relays have been developed to use these symmetrical components in analyzing all faults types. Engineers found that relays based on positive sequence measurement give good indications for the balance fault, and the zero sequence relays have accurately indicated the ground faults. However, with the protection devices developments, negative sequence relays became more familiar to the power system community. Relays based on negative sequence component quantities can detect all unbalanced fault types, especially line-line faults which are considered as the common events in power systems, in addition to more advantages will be reviewed later in this paper.

\section{NEGATIVE SEQUENCE PROTECTION OVERVIEW}

Negative sequence Protection (NSP) is a protection scheme used to protect the power system element by means of negative sequence component. It was first introduced in protection as a negative sequenced overcurrent relay around 1950s [4]. Since then, researchers and protection engineers have noted the high sensitivity of the NSP towards unbalanced faults. NSP has been used with almost all the power system elements but especially generators and motors [5]. It also has been adapted for use with mainly overcurrent, differential and distance relays. Reference [4] described the analysis of an electrical system with all types of asymmetrical faults (line-ground, line-line, and line-line-ground). After analyzing the unbalanced system to its equivalent sequence components, it has been found that the negative sequence component gives a good indication for the ground type faults as does the zero sequence. However, the negative sequence component gives a better indication of line-line faults, where these faults are one of the main causes of the negative sequence component in the system. NSPs are designed to have an embedded filter circuit that only activates when unsymmetrical faults occur. This negative sequence filter circuit was difficult to apply with earlier protection devices but, with numerical relays, it became more simple. Fig. 1 shows an example of a negative sequence filter with an electromechanical relay [6].

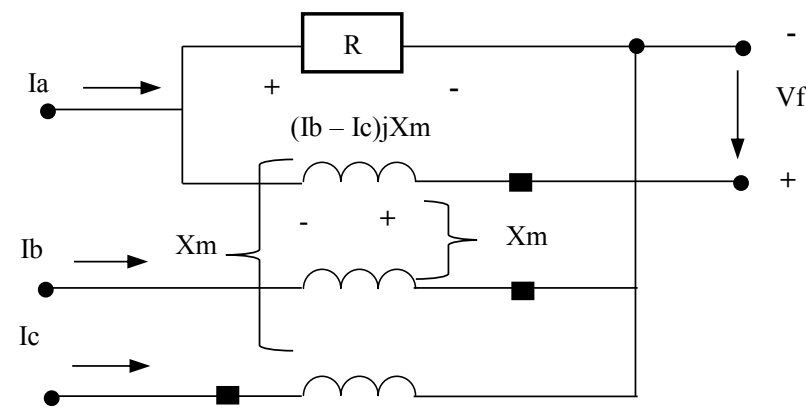

Fig. 1. Negative-sequence filter in an electromechanical relay, retrieved from [6]

This design shows that the output of the filter is proportional to the three-phase input current, this filter is only responsive to the negative component with nil output of positive (the balanced condition) and zero components. A zero component filter was then developed and used for its 
effectiveness with ground fault, faults that occur between one or two lines and the ground. It is much simpler than the negative one because the sequence element has zero phase shifting. The revolution in power electronic technology has resulted in making relays more functional and able to analyze all sequence components accurately and easily.

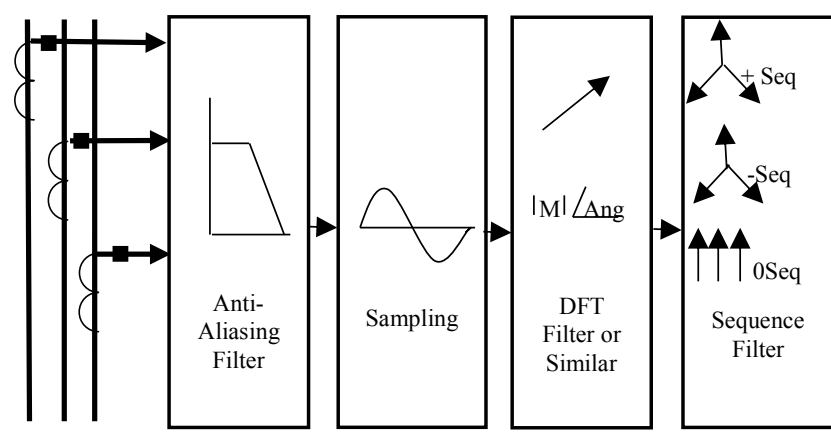

Fig. 2. Sequence quantities in a numerical relay, retrieved from [6]

In power system, basically, relays are connected to current transformers (CT) or voltage transformer (VT) to provide a suitable value of voltage and current measurement for the protection device. Reference [7] illustrated that when the secondary circuit of the instrument device failed due to a broken neutral, the filter in the relay will not be able to measure the zero sequence component. However, the negative sequence will be measured and will activate the relay trip. In addition, the negative sequence component measurement is not affected by the mutually coupled line currents [5]. Negative sequence-based protection made a very useful addition to the already established protection techniques when it became easier to implement in the modern digital relays.

\section{A. Negative sequence protection for generator protection}

Negative sequence protection is needed for generators because of probable damage caused by unbalanced currents resulting from unbalanced load conditions. ANSI standards allow negative sequence current $I_{2}$ of $5-10 \%$ in generators with short time limits defined as $\left(I_{2}\right)^{2} \mathrm{t}$ [7]. Unbalanced currents causes the negative sequence component of current in the stator of the generator to rise [8]. These unbalanced conditions result in heating of the rotor that leads to mechanical stress on the rotating components due to induced double frequency currents caused by these negative sequence currents [7]. The negative sequence current produces an additional counter flux that rotates at synchronous speed in the opposite direction to the rotor. As a solution for that possible damage, a static negative sequence time overcurrent relay was developed to directly sense the negative sequence current component that might cause overheating in the generator rotor [4]. Reference [7] showed a detailed structure of the negative sequence overcurrent relay. The negative sequence current components have serious effects on the supersynchronous resonance of turbine blades, so that [8] suggested using Static VAr Compensator (SVC) to reduce or block the negative sequence current at generator terminals. This method could be considered the most suitable via blocking the negative sequence current; as long as the compensation circuit is guaranteed to operate only during unbalanced events and with very well designed harmonic filters. Reference [9] tried to use a directional relay to detect generators' inner asymmetrical fault based on negative sequence voltage and current components. The research showed it to be a highly sensitive and secure scheme with only theoretical solution for the harmonic and oscillation issue.

The revolution of renewable energy for reducing the greenhouse gas emissions led the power system community to introduce new generation techniques such as wind turbines as the most promising renewable energy technology. These new generators have challenges regarding their security, where significantly rapid protection is needed to avoid destroying the converters' elements under fault conditions and to avoid islanding implications [10]. Reference [11] shows that wind turbines need a scheme to control the negative-sequence efforts. The highest negative sequence quantity produced in a wind turbine generator usually is during line-line faults, which are considered as the most serious scenario regarding the fault ride-through code standards.

\section{B. Negative sequence protection for transformers protection}

Transformers, like any other element in the power system, need a sensitive fault detection device. A transformer's health is usually affected by loading beyond nameplate rating, external faults, over excitation due to eddy currents and external system conditions. All these result in rising temperature of the windings and coils leading to weakening the integrity of the winding or severe damage in some cases.

Differential relays are commonly used as an overall protection device for transformers. These relays are designed to solve the problems of second harmonic generated by inrush current and CT saturation, either by adding an instantaneously tripping differential current to the original harmonic restraint differential protection [10] or by cancelling the second harmonic restraint by a low voltage criteria [12]. However, [11] states that the differential relay fails to detect a fault in about 3\% of fault events due to harmonic distortion caused by fault currents and by CT saturation under fault, so that many researchers propose methods based on symmetrical components to overcome such issues.

The negative sequence element according to [13]-[15] is sensitive, secure and independent of load. It enhances the discrimination reliability between internal (in phase) and external (out of phase) faults of the existing differential protection even with low-level turn-to-turn faults. This negative sequence filter is embedded with transformer's protection scheme and is hardly affected by harmonic distortion under the DC bias of the protected transformer [16]. Reference [14] also showed that using negative sequence current based differential overrides the long delay time and unwanted operation under external faults whilst increasing the sensitivity to internal faults. Reference [14] indicated that embedded negative elements are able to detect faults across up to $10 \%$ of transformer winding turns, while the traditional relay can detect faults only for $2 \%$ of the transformer windings.

However, [15] shows that the differential relay based NSP is a little bit less sensitive than that based on space vector protection in $14 \%$ of the internal fault (low level fault) scenario, while both protection types are similar regarding heavy faults. 
Reference [10] suggested using a negative sequence power-based differential protection to cancel the second harmonic generated during internal faults and avoid the long delay time; this cannot be achieved with traditional differential relays. Reference [16] suggested an approach based on calculating the difference between the primary of CT1 and secondary of CT 2 current negative component. This method is less affected by CT saturation, and magnetizing inrush.

Reference [17] discussed the effect of a single phasing fault on the primary of transformers used to serve rural loads that are protected by fuses. The latter suggests using negative sequence-based overvoltage relays to detect the low voltage drop occurring due to a fuse blowing in the primary; this is quite similar to the technology used with large synchronous machines in industrial applications. This type of fault will not cause the feeders' circuit breakers to trip unless the unbalance current is enough to make the ground relays operate.

NSP relays highly advanced the principle of transformer protection to solve or at least to mitigate many reliability problems faced by power transformers, especially low-level turn-to-turn faults.

\section{Negative sequence protection in distribution network protection}

Recently there have been some radical changes in distribution networks due to load types and the transition to smart grid in addition to the new technology of distributed generation (DG). These changes together began affecting the use of conventional overcurrent relays because of diminished fault current levels.

NSP-based overcurrent relays (OCR) provide faster and more sensitive operation than the ordinary phase overcurrent elements for the same disturbance that might occur in the network. Reference [18] suggested using NSP depends on current measurement but, unlike the traditional OCR, the pickup current is less than load levels. Coordinating the modified microprocessors relays (as a negative sequence OCR) with the conventional ones became a necessity. Reference [18] set out some guidelines for coordination with downstream relays in a radial distribution network. These guidelines are based on considering the negative sequence overcurrent element equivalent to the conventional one. Then the thresholds are set based on that assumption. The current setting of the traditional OCR then should multiply by $\sqrt{3}$ to convert it to the negative sequence quantity in terms of $3 I_{2}$. The time delay, curve type and time dial set of the traditional OCR remains the same with no need for any conversion factor.

Reference [19] suggested to measure the ratio of the negative sequence current to the positive component to identify faults in radial feeders; the study showed that the ratio value would increase when the fault is closer to the measuring device.

Another advantage of using NSP in the modern network is anti-islanding protection of DGs in the system due to its large threshold window compared to conventional methods used previously as detailed in [20]. Reference [21] discussed using the negative sequence voltage to detect an islanding situation. With this method, negative sequence voltage damping pattern will be needed to reduce the capacitor and parallel line switching issues. This negative sequence technique for DG islanding detection is more reliable when negative sequence voltage will be considered during islanding period. Reference [20] used negative sequence impedance for anti-islanding protection in less than 4 cycles with a wide impedance threshold window. Reference [22] emphasized the idea that pilot differential protection is the best with respect to feeder protection in a network with embedded DGs, however, the reliability of this method could be enhanced by using a negative sequence directional element with it. All above references make suggestions on how to deal with short circuit fault current using NSP.

Reference [17] demonstrated a method on how to catch an open circuit fault based on voltage negative sequence measurement instead of relying on customer complaints. This method detects the voltage abnormality in two or three phases that the relays downstream are unable to sense due to absence of current. This is applicable to broken conductor and transformer blown fuse issues as mentioned earlier.

The inverter-interfaced DGs in distribution networks has another issue regarding protection due to their current limitation characteristic. Thus, the traditional relays will not operate accurately for their limited fault level. To improve inverter-interfaced DG protection scheme [23] suggests using a negative-sequence control structure for inverter-interfaced DGs using NSP.

\section{Negative sequence techniques with transmission lines}

TLs are considered as the one element of the power system components having the highest fault occurrence due to their exposure to the environmental events, for instance, lightning, storms, and insulation failure. Many protection schemes used with this network are based on considering factors such as the model of the line, the instrument transformers, communication, and whether the measurements synchronize or not. Reference [24] illustrated the progress of the protection technologies used with TL, and the algorithms usually used or proposed for faults' precise location.

Zero-sequence based differential relays are usually used as a backup protection for the TL due to their accuracy in detecting ground faults, and the ability to be fed to a measuring unit directly. Technological progress in microprocessor relays offered the chance to have a more reliable protection scheme using embedded negative sequence filters in relays.

The negative sequence component is quite similar to the zero component regarding detecting system abnormality. However, it seems that differential protection based on the negative sequence component has more advantages over the one based on zero component, where the former is sensitive to all unsymmetrical faults types while the latter senses only ground faults [25]. The other advantage of the negative sequence current components over zero sequence current component is that mutually coupled parallel line currents do not influence the measurement and only the three-phase currents are used as inputs (the neutral current is not needed) [5]. Reference [14] highlighted the advantage of having a line differential protection with a negative element, where the sensitivity of the differential relay increases due to removing the load effect from the differential relay constraint signals. In addition it is less affected by the line charging current.

Distance relaying is the most common used relay with high and extra high voltage (EHV) transmission and sub- 
transmission lines due to the high-speed fault clearance compared to differential and overcurrent relays. This relay is based on measuring the impedance, which is proportional to the electrical distance between relay and fault location; and then compares it with the threshold impedance to decide which switch to trip. Under heavy load conditions, the conventional distance relay may not be able to identify the faulty zone. It became a necessity to find a solution that is not affected by load conditions, long distance lines, large capacitive currents and line distributed parameters. Reference [26] reported that long distance factor with bulk lines requirements can be mitigated by using relays based on traveling wave, and suggested the idea of using negative sequence current components distance relay based on Bergeron line model. The suggested technique will operate only under unbalanced system faults irrespective of the load conditions and system fluctuations with a high reliability both inside and outside protection zones.

With the development of communication strategies between protection devices, the pilot protection based on negative sequence components is used widely. However, this technology faced some issues regarding the open-phase faults in EHV TLs. Reference [27] studied the effectiveness of the pilot protection with an open circuit fault in EHV TLs. This algorithm was based on resolving load voltage into negative voltage components when an open phase fault occurs. This algorithm improved the sensitivity and selectivity of the protection device to be used as TL main protection.

There have not been so many papers published on using negative sequence current protection techniques for TL in the last 20 years. However, there has been some research done on using negative sequence elements algorithms to locate faults in transmission networks [28]-[30]. Most of the proposed methods were based on using negative sequence voltage in a fault location algorithm to avoid causes mentioned earlier, assuming that the fault was already detected. Reference [31] suggested using synchronized negative voltage components from two-ended double line circuits by comparing their magnitude at both ends of the line. The main effective feature of this method was that there was no need to do any pre-fault calculation, fault classification or fault resistance estimation. The author of [31] tried to modify his algorithm to be able to detect the fault using voltage and current measurements and to locate the fault using only the negative voltage component [28]. Reference [29] developed the previous algorithm to be suitable to a lumped and PI line models, but this time with unsynchronized negative voltage measurement and the shunt capacitance calculated along the TL. The latter algorithm still gives a reliable result with less percentage error. Reference [30] examined using the same algorithm of negative voltage elements with three different scenarios: single circuit line, single circuit linked to double circuit line and the double circuit line respectively in a Tconnection network. The latter study tried to overcome the issues of locating a fault precisely in a three terminal, $\mathrm{T}$ connected system, with synchronized or unsynchronized measurements and under different fault resistances. The error percentage of this method is $85 \%$ less than those in [32], [33].

\section{TRANSMISSION LINE PROTECTION CHALLENGES}

In spite of all the aforementioned, TL network protection still has some challenges due to factors, such as:

\section{A. DC offset and CT saturation}

Most of the protection relays used with TL use phasor quantities of voltage and current, where their calculation could be complex due to exponential decay offset DC. This will lead to the fault current being unsymmetrical during the first few cycles. The extent of this asymmetry depends on the fault point on the voltage waveform. Before DC offset decays and when fault current passes through, CT saturation will occur and when DC offset disappears the CT will return to its normal operation condition.

Since most of the relays used with TLs are current-based, the CT saturation current will affect the current magnitude and may cause a tripping delay time as mentioned earlier. CTs, like any transformer, have two coils; primary and secondary. The ratio between the two coils' windings of a CT might result in saturation current in the secondary side of the transformer during heavy fault event; this in turn leads to relay maloperation [34]. The high fault current prevents the CT from sending an appropriate signal to the relay, where the relay responds to only low proportional secondary current during acute faults. Although upstream relays used in the TL network with a high $\mathrm{CT}$ ratio might measure the fault current more accurately and trip, the selectivity of the protected zone will be lost due to the low ratio CT saturation [35].

Many algorithms and methods have been proposed by academic research and industry to prevent or mitigate the CT saturation issue. The first idea is to increase the size of the secondary core to be able to handle high fault current, but this solution is unreliable due to the high cost of manufacture and of replacing all existing CTs. Reference [36] suggested injecting a secondary circuit exponential decaying DC, but this did not work for all decaying DC levels. Reference [37] argued that a compensation algorithm could solve this problem, where the algorithm will sense the saturation as soon as possible and then estimate the magnetization current or the core flux during the fault. The exact secondary current will then be calculated by combining the measured secondary current and the magnetization current. Reference [38] noted that this compensation algorithm method has a negative side that assumes "the residual flux to be zero or known". However, [39] discussed using the compensation algorithm with neural networks by providing a CT inverse function. Reference [8] argued that the neutral CT with its lower ratio (to calculate zero and negative sequence current) provides greater sensitivity than higher ratio phase CTs.

\section{B. Renewable energy source control effects}

Most of the new generation mix in power systems are renewables such as wind turbines and photovoltaic solar cells connected in both the generation and distribution sides of the network. These resources are usually interfaced by power electronic elements. The low thermal inertia of these elements results in limiting the current flow through them. This limit made the inverter-based resources (IBR) contribution to fault current very low compared to conventional AC synchronous generators. The high penetration of these IBRs in distribution network caused a topical issue from the protection perspective, since the majority of the protection schemes are designed to activate under high fault current [40].

This issue is not associated only with distribution side of the system due to microgrid development, it also affects TL connected to large-scale wind turbine farms and solar cell plants. Many countries have introduced standards and codes to improve their power system reliability. Reference [41] 
discussed the effect of the wind farm control devices on negative sequence current during fault events and hence the protection device accuracy. Reference [42] reported that the controls of wind turbines might reduce or even eliminate the effect of the fault negative current component to comply with grid code, which interferes with protection device functionality. The design of inverter control could distort the current waveform causing high harmonic distortion. In comparison to traditional synchronous generators, the harmonics resulting from IBRs may result in unpredicted phase shifts both in angle and magnitude. Many algorithms have been proposed to use with different protection relays such as using distance relay with an inverse time admittance characteristic [43]. This approach had the disadvantage of having a long period tripping time in addition to the reduced accuracy due to the current transient and DC decaying magnitude when a fault occurred. References [44]-[46] proposed using OCR based on symmetrical components with a directional element. The difficulty of considering all contingencies and scenarios for the relay settings made overcurrent schemes unsuitable for distribution networks anymore.

\section{Zero-sequence mutual coupling}

This might be considered to be the most common problem in double circuit TL. TLs might be parallel, supported by the same tower or may share the same side of the way. All these situations could cause some mutual coupling between lines affecting the ground current measured by a protective relay. As a result, apparent impedance calculations will be affected by the current flowing in the parallel TL [47].

Since most of the modern protection relays are based on symmetrical component in fault analysis, it is worth mentioning that the positive and negative sequences coupling between parallel lines have a very small effect, almost negligible, on protection relays' operation. Zero sequence mutual coupling, on the other hand, has a great effect on the parallel lines or a double circuit line from protection perspective since its amount is equal to $70 \%$ of the line zero impedance [48]. The ground directional elements of the overcurrent and distance protection relays are the most affected by this magnetic phenomenon as they deal with zero sequence directional elements.

Reference [49] explained that having a negative sequence based directional element with protection relay instead of zero sequence ones helps to eliminate the mutual coupling effect on the relay's operation. Differential relays showed a good sensitivity to these faults regardless of the zero mutual coupling impedance particularly with double circuit TLs having jumper across the similar phases [49], [50] but it required a fast communication link and a remote backup protection, which is quite difficult [47].

TABLE I: references regarding paper's items

\begin{tabular}{|l|c|}
\hline \multicolumn{1}{|c|}{ Item } & References \\
\hline NSP with rotating machines & {$[7]-[11]$} \\
\hline NSP with transformers & {$[12]-[17]$} \\
\hline NSP with distribution feeders & {$[18]-[23]$} \\
\hline NSP with transmission lines & {$[24]-[33]$} \\
\hline Current transformers issue & {$[34]-[39]$} \\
\hline $\begin{array}{l}\text { Renewable energy source control devices } \\
\text { effect issue }\end{array}$ & {$[40]-[46]$} \\
\hline
\end{tabular}

\begin{tabular}{|l|r|}
\hline Zero current mutual coupling issue & [47-50]
\end{tabular}

\section{CONCLUSION}

Most of the traditional protection techniques used with high voltage TLs are accurate to some extent, especially with the implementation of digital relays and symmetrical component protection practice. However, issues start to develop due to the high penetration of renewable energy sources in the power system. In this paper, different schemes based on the negative sequence component were reviewed. Some issues and challenges of using current-based protection schemes for transmission networks must be solved. It is highly recommended to modify the settings of the existing protection schemes or devise a new protection scheme to improve the fault detection and location accuracy to save the system equipment from damage and to reduce power interruptions.

\section{ACKNOWLEDGMENT}

The authors acknowledge funding support to carry out this research from University of Auckland Doctoral Scholarship; University of Auckland under Faculty Research Development Fund (FRDF) Grant 370953; and, Ministry of Business, Innovation and Employment, New Zealand's National Science Challenge-Resilience to Nature's Challenge and in particular the project on Electricity Distribution Resilience Framework informed by West Coast Alpine Fault Scenario.

\section{REFERENCES}

[1] S. U. Karpe and M. N. Kalgunde, "Power system backup protection in smart grid using synchronized PMU," Int. Conf. Signal Process. Commun. Power Embed. Syst. SCOPES 2016 - Proc., pp. 1139-1144, 2017.

[2] S. Marx, D. Bender, and B. P. Administration, "An introduction to symmetrical components, system modeling and fault calculation," 2013.

[3] C. L. Fortescue, "Method of symmetrical co-ordinates applied to the solution of polyphase networks," Trans. Am. Inst. Electr. Eng., vol. XXXVII, no. 2, pp. 1027-1140, 1918.

[4] D. J. Graham, P. G. Brown, and R. L. Winchester, "Generator protection with a new static negative sequence relay," IEEE Trans. Power Appar. Syst., vol. 94, no. 4, pp. 1208-1213, 1975.

[5] M. M. Aman, G. Bin Jasmon, H. Bin Mokhlis, Q. A. Khan, A. H. B. A. Bakar, and M. Karimi, "Modeling and simulation of digital frequency relay for generator protection," PECon 2012 - 2012 IEEE Int. Conf. Power Energy, pp. 701-706, 2012.

[6] F. Calero, "Rebirth of negative-sequence quantities in protective relaying with microprocessor-based relays," 57th Annu. Conf. Prot. Relay Eng., pp. 190-219, 2004.

[7] B. Lewis, and T. Domin," Protective relaying principles and applications," Taylor \& Francis Group, LLC, United States of America, third edition, 2007.

[8] W. C. Morris, and L. E. Goff, "Negative phase sequence overcurrent relay for generator protection," Transactions of the American Institute of Electrical Engineers. Part III: Power Apparatus and Systems, vol.72, no.4, pp.615-620, 1953.

[9] L. Shiming, C. Deshu, Y.Xianggen, and T. Huiliang, "Fault component negative-sequence energy direction protection for generators," Power Engineering Society Winter Meeting, Singapore, vol. 3, no. I, pp. 1878-1882, 2000

[10] K. Tian and P. Liu, "Improved operation of differential protection of power transformers for internal faults based on negative sequence power," International Conference on Energy Management and Power Delivery, Singapore, pp. 422-425, 1998.

[11] I. Brncic, Z. Gajic, and T. Einarsson, "Transformer differential protection improved by implementation of negative-sequence currents," ABB. Power Technologies, Sweden.

[12] P. Liu, D. Chen, Y. Guo, O. P. Malik, and G. S. Hope, "Improved 
operation of differential protection of power transformers for internal faults," IEEE Transactions on Power Delivery, vol. 7, no. 4, pp. 1912 1919,1992

[13] I. A. Rizvi and G. Reeser, "Using symmetrical components for interna external fault discrimination in differential protection schemes," 66th Annu. Conf. Prot. Relay Eng., pp. 68-79, 2013.

[14] B. Kasztenny, N. Fischer, and H. J. Altuve, "Negative-sequence differential protection - principles, sensitivity, and security," 68th Annual Conference for Protective Relay Engineers, USA. pp. 364 378, 2015.

[15] L. M. R. Oliveira and A. J. M. Cardoso, "Comparing power transformer turn-to-turn faults protection methods: negative sequence component versus space-vector algorithms," IEEE Transactions on Industry Applications, vol. 53, no. 3, pp. 2817-2825, 2017.

[16] W. X. Li, L. G. Liu, T. Zheng, G. Huang, and H. Shi, "Research on effects of transformer DC Bias on negative sequence protection," International Conference on Advanced Power System Automation and Protection, vol. 2, pp. 1458-1463, 2011.

[17] S. K. Lau and S. K. Ho, "Open-circuit fault detection in distribution overhead power supply network," Journal of International Council on Electrical Engineering, vol. 7, no. 1, pp. 269-275, 2017.

[18] A. F. Elneweihi, E. O. Schweitzer, and M. W. Feltis, "Negativesequence overcurrent element application and coordination in distribution protection," IEEE Transactions on Power Delivery, vol. 8, no. 3, pp. 915-924, 1993

[19] C. I. Ciontea, C. L. Bak, F. Blaabjerg, K. K. Madsen, and C. H Sterregaard, "A feeder protection method against the phase-phase fault using symmetrical components," IEEE Electric Ship Technologies Symposium, USA, pp. 56-63, 2017.

[20] M. Wrinch, J. Martí, and M. Nagpal, "Negative sequence impedance based islanding detection for distributed generation (NSIID)," Electric Power Conference, Canada, pp. 1-6, 2008.

[21] A. Helmzadeh, J. Sadeh, and O. A. Musavi, "Anti-islanding protection of distributed generation resources using negative sequence component of voltage," International Conference on Power Engineering, Energy and Electrical Drives, Portugal, pp. 440-443, 2007.

[22] G. G. Karady and P. Mandava, "Pilot directional protection using negative sequence directional element," Power Systems Conference, Clemson University, USA, vol. 12, no. 1, pp. 1-4, 2014

[23] H. Shao, H. Zhu, R. Lin, G. Xu, Q. Yu, and C. He, "Analysis on IIDG's negative-sequence current upon asymmetric fault," International Conference on Electricity Distribution, Chin, 2016.

[24] C. Poongothai, "A review on HVDC protection system," IEEE International Conference on Circuits and systems, India, pp. 134-139, 2017.

[25] N. Zhang, X. Dong, Z. Bo, and A. Klimek, "Performance Comparison of Current Differential Protection Scheme Based On Symmetrical Components," 42nd International Universities Power Engineering Conference, UK, pp. 305-309, 2007.

[26] L. Bin, H. Jialil, and B. Zhiqian, "The negative-sequence distance protection for UHV long distance transmission line," IET 9th International Conference on Developments in Power System Protection, UK, pp. 632-637, 2008

[27] B. Wang, X. Dong, Z. Bo, and A. Klimek, "Negative-sequence pilo protection with applications in open-phase transmission lines," IEEE Transactions on Power Delivery, vol. 25, no. 3, pp. 1306-1313, 2010.

[28] B. Mahamedi and J. G. Zhu, "Unsynchronized fault location based on the negative-sequence voltage magnitude for double-circuit transmission lines," IEEE Transactions on Power Delivery, vol. 29, no. 4, pp. 1901-1908, 2014.

[29] A. Amireddy and K. K. Pedapenki, "Negative sequence voltage magnitude based unsynchronized fault location on three terminal lines," International Journal of Innovative Research in Electrical, Electronics, Instrumentation and Control Engineering, vol. 5, no. 7, pp 61-67, 2017

[30] G. Yang, N. Tai, X.Zheng, and C. Wang, "Fault location for threeterminal transmission lines using fault voltage sequence components,", Australian Journal of Electrical and Electronics Engineering, vol.11, no. 2, pp. 265-273, 2014

[31] S. M. Brahma and A. A. Girgis, "Fault location on a transmission line using synchronized voltage measurements," IEEE Transactions on Power Delivery, vol. 19, no. 4, pp. 1619-1622, 2004.

[32] J. Izykowski, E. Rosolowski, M. M. Saha, M. Fulczyk, and P. Balcerek, "A fault-location method for application with current differential relays of three-terminal lines," IEEE Transactions on Power Delivery, vol. 22, no. 4, pp. 2099-2107, 2007.

[33] Y. H. Lin, C. W. Liu, and C. S. Yu, "A new fault locator for threeterminal transmission lines - Using two-terminal synchronized voltage and current phasors," IEEE Transactions on Power Delivery, vol. 17, no. 2, pp. 452-459, 2002.

[34] K. Wannous and P. Toman, "The impact of current transformer saturation on the distance protection," 17th International Scientific Conference on Electric Power Engineering, pp. 1-5, 2016.

[35] S. Holst, I. Brnčič, D. Shearer, R. Mangelred, and K. Koreman, "Problems and solutions for ac transmission line protection under extreme conditions caused by very long HVDC cables," Cigre, Study Committee B5 Colloquium, Spain, pp. 1-8, 2007.

[36] P. Jena, A. Pradhan, "Reducing current transformer saturation effect in phasor measurement unit," International Transactions on Electrical Energy Systems, vol. 26, no. 7, pp. 1397-1407, 2015.

[37] H.-W. Lee, Y.-C. Kang, S.-I. Jang, and Y.-G. Kim, "Distance relay suitable for use with a measurement type current transformer," IEEE Lausanne Power Tech, pp. 1176-1181, 2007.

[38] B. Kasztenny, J. Mazereeuw, K. Jones, "CT saturation in industrial applications - analysis and application guidelines," GE Multilin, pp. 386-417, 2007.

[39] S. Bittanti, F. A. Cuzzola, F. Lorito, and G. Poncia, "Compensation of nonlinearities in a current transformer for the reconstruction of the primary current," IEEE Transactions on Control Systems Technology, vol. 9 , no. 4 , pp. 565-573, 2001.

[40] IEEE PES-TR68, "Impact of inverter based generation on bulk power system dynamics and short-circuit performance," July, 2018.

[41] H. Geng, C. Liu, and G. Yang, "LVRT capability of DFIG-based WECS under asymmetrical grid fault condition," IEEE Transactions on Industrial Electronics, vol. 60, no. 6, pp. 2495-2509, 2013.

[42] I. Erlich, T. Neumann, F. Shewarega, P. Schegner, and J. Meyer, "Wind turbine negative sequence current control and its effect on power system protection," IEEE Power \& Energy Society General Meeting., no. Type 3, pp. 1-5, 2013

[43] M. Dewadasa, R. Majumder, A. Ghosh, and G. Ledwich, "Control and protection of a microgrid with converter interfaced micro sources," International Conference on Power Systems, pp. 25-30, 2009.

[44] B. Li, Y. Li, Z. Bo, and A. Klimek, "Design of protection and control scheme for microgrid systems," 44th IEEE International Universities Power Engineering Conference, pp. 444-448, 2009.

[45] M. A. Zamani, T. S. Sidhu, and A. Yazdani, "A protection strategy and microprocessor-based relay for low-voltage microgrids," IEEE Transactions on Power Delivery, vol. 26, no. 3, pp. 1873-1883, 2011.

[46] E. Sortomme, S. S. Venkata, and J. Mitra, "Microgrid protection using communication-assisted digital relays," IEEE Transactions on Power Delivery, vol. 25, no. 4, pp. 2789-2796, 2010.

[47] A. Apostolov, D. Tholomier, S. Sambasivan, and S. Richards, "Protection of double circuit transmission lines," 60th Annual Conference for Protective Relay Engineers, pp. 85-101, 2007.

[48] V. H. Makwana, and B. R. Bhalja, "A new adaptive distance relaying scheme for mutually coupled series-compensated parallel transmission lines during intercircuit faults," IEEE Transactions on Power Delivery, vol. 26 , no. 4, pp. 2726-2734, 2011

[49] D. A. Tziouvaras, H. J. Altuve, and F. Calero, "Protecting mutually coupled transmission lines: Challenges and solutions," 67th Annual Conference for Protective Relay Engineers, pp. 30-49, 2014.

[50] J. Roberts, D. Tziouvaras, G. Benmouyal, and H. Altuve, "The effect of multiprinciple line protection on dependability and security," 54th Annual Conference for Protective Relay Engineers, 2001. 